version June 20, 2018: fm

\title{
Searching for $\gamma$-ray blazar candidates among the unidentified INTEGRAL sources
}

\author{
F. Massaro ${ }^{1}$, A. Paggi ${ }^{2}$, R. D’Abrusco ${ }^{2}$ \& G. Tosti ${ }^{3,4}$. \\ SLAC National Laboratory and Kavli Institute for Particle Astrophysics and Cosmology, 2575 \\ Sand Hill Road, Menlo Park, CA 94025 \\ Harvard - Smithsonian Astrophysical Observatory, 60 Garden Street, Cambridge, MA 02138 \\ Dipartimento di Fisica, Università degli Studi di Perugia, 06123 Perugia, Italy \\ Istituto Nazionale di Fisica Nucleare, Sezione di Perugia, 06123 Perugia, Italy
}

\begin{abstract}
The identification of low-energy counterparts for $\gamma$-ray sources is one of the biggest challenge in modern $\gamma$-ray astronomy. Recently, we developed and successfully applied a new association method to recognize $\gamma$-ray blazar candidates that could be possible counterparts for the unidentified $\gamma$-ray sources above $100 \mathrm{MeV}$ in the second Fermi Large Area Telescope (LAT) catalog (2FGL). This method is based on the Infrared (IR) colors of the recent Wide-Field Infrared Survey Explorer (WISE) all-sky survey. In this letter we applied our new association method to the case of unidentified INTEGRAL sources (UISs) listed in the fourth soft gamma-ray source catalog (4IC). Only 86 UISs out of the 113 can be analyzed, due to the sky coverage of the WISE Preliminary data release. Among these 86 UISs, we found that 18 appear to have a $\gamma$-ray blazar candidate within their positional error region. Finally, we analyzed the Swift archival data available for 10 out these $18 \gamma$-ray blazar candidates, and we found that 7 out of 10 are clearly detected in soft X-rays and/or in the optical-ultraviolet band. We cannot confirm the associations between the UISs and the selected $\gamma$-ray blazar candidates due to the discrepancies between the INTEGRAL and the soft X-ray spectra. However, the discovery of the soft X-ray counterparts for the selected $\gamma$-ray blazar candidates adds an important clue to help understand their origin and to confirm their blazar nature.
\end{abstract}

Subject headings: galaxies: active - galaxies: BL Lacertae objects - radiation mechanisms: non-thermal 


\section{Introduction}

One of the main scientific objectives of the INTEGRAL mission is performing a survey of the sky in a mostly unexplored region of the electromagnetic spectrum: from the hard X-ray to the soft $\gamma$-ray band. Since its launch in October 2002, INTEGRAL has used the unprecedented imaging capabilities of IBIS (Imager on Board INTEGRAL Spacecraft: Ubertini et al. 2003) coupled with those of ISGRI (INTEGRAL Soft Gamma-Ray Imager; Lebrun et al. 2003). Combining data from these two instruments, it is possible to generate images of the sky with a 12 arcmin (Full Width Half Maximum, FWHM) resolution with typical source location accuracy of $\sim 1-3$ arcmin over a $\sim 19$ degree (FWHM) field of view in the energy range 17-1000 keV.

The fourth soft $\gamma$-ray source catalog 1 (Bird et al. 2010) (hereinafter 4IC) obtained with the IBIS $\gamma$-ray imager on board the INTEGRAL satellite lists 723 hard X-ray/soft $\gamma$-ray sources. In particular, the 4IC substantially increased the extragalactic sky coverage including both transients and faint persistent objects that can only be revealed with long exposure observations (Bird et al. 2010).

Several observations at low energies have been already performed to decrease the number of the unidentified INTEGRAL objects (UISs) (see e.g., Masetti et al. 2008; Masetti et al. 2009; Masetti et al. 2010, for optical and X-ray observations of UISs); however, a considerable fraction of the 4IC sources are still completely unidentified. According to the 4IC, there are 113 UISs, corresponding to about $16 \%$ of the whole catalog, and 178 other sources have uncertain classification. The largest fraction (i.e., 35\%) of the associated INTEGRAL sources are Active Galactic Nuclei (AGN), compared to $31 \%$ identified as Galactic sources (Bird et al. 2010). For comparison, the 58-month catalog of observations with the BAT hard X-ray detector 2 on board the Swift observatory, lists 1092 objects detected in the 14-195 keV energy range, with 86 unidentified hard X-ray sources listed (Cusumano et al. 2010; Baumgartner et al. 2010).

Recently, using the WISE all-sky IR survey, we discovered that blazars, the largest known $\gamma$ ray class of AGN, can be separated from other extragalactic sources using IR colors (Massaro et al. 2011a, hereinafter Paper I). We used the magnitudes of the recent WISE IR all-sky survey performed at $3.4,4.6,12$, and $22 \mu \mathrm{m}$ with an angular resolution of $6.1,6.4,6.5 \& 12.0$ arcsec and with $5 \sigma$ point source sensitivities achieving $0.08,0.11,1$ and $6 \mathrm{mJy}$, in unconfused regions on the ecliptic, respectively. The absolute (radial) differences between WISE source-peaks and "true" astrometric positions anywhere on the sky are no larger than $\sim 0.50,0.26,0.26$, and 1.4 arcsec in the four WISE bands, respectively (Cutri et al. 2011) 3 .

\footnotetext{
${ }^{1}$ http://irfu.cea.fr/Sap/IGR-Sources/

${ }^{2}$ http://heasarc.nasa.gov/docs/swift/results/bs58mon/

${ }^{3}$ http://wise2.ipac.caltech.edu/docs/release/prelim/expsup/sec2_3g.html
} 
Moreover, we investigated a sample of blazars detected by WISE and associated with FermiLAT sources to characterize their IR- $\gamma$-ray properties (D'Abrusco et al. 2012, hereinafter Paper II). This was the first step to develop a new association method for the unidentified $\gamma$-ray sources, able to recognize if there is a $\gamma$-ray blazar candidate within their positional error region (Massaro et al. 2012a; Massaro et al. 2012b, hereinafter Paper III and Paper IV, respectively). With this new IR diagnostic tool, we searched for $\gamma$-ray blazar candidates within the unidentified $\gamma$-ray source sample of the 2FGL $\gamma$-ray catalog, and for the first time we have been able to provide a candidate counterpart for 187 out of 313 unidentified $\gamma$-ray sources analyzed, having the same IR properties as the $\gamma$-ray emitting blazars (see Paper IV).

In this letter, we apply this association procedure to test whether there is a possible $\gamma$-ray blazar candidate for the UISs using their WISE IR colors. For the selected $\gamma$-ray blazar candidates, we also search Swift pointed observations for the presence of an optical-UV and/or X-ray counterpart. This letter is organized as follows: in Section 2 we describe the UIS sample selected for our investigation; in Section 3 we illustrate the basic details of our new association method, that, in Section 4 , we apply to the UISs. Section 5 is devoted to the optical-UV-X-ray counterparts in the Swift observations available. Our results are discussed in Section 6 .

(1) with uncertain classification.

In this letter, we only considered the 86 UISs out of 113 listed in the 4IC that lie in the portion of the sky covered by the WISE Preliminary Source catalog.

In the 4IC there are 113 sources that are completely unidentified (i.e., Type =?, according to Table 3 in Bird et al. 2010), while there are 97 sources that are indicated as unidentified transients (i.e., Type =?,T, according to Table 3 in Bird et al. 2010). In addition, within the 4IC, there are also 32 AGN sources of uncertain type (i.e., Type =AGN? Bird et al. 2010) and another 49 objects 
lie in the subregion of the $W G S$ occupied by the BZBs and BZQs using the $s_{b}$ and $s_{q}$ parameters separately (Paper III).

In Paper IV, we presented the association method based on the WGS parametrization. For each unidentified $\gamma$-ray source we defined the searching region corresponding to a circular region of radius $R$ equal to the semi-major axis of the elliptical source location region at $99.999 \%$ confidence level, centered on the $\gamma$-ray position given in the 2FGL catalog(Nolan et al. 2012).

We calculate the IR colors for every WISE source that lies within the searching region as well as their $s_{b}$ and $s_{q}$ parameters. Given the distributions of generic WISE sources in random regions of the sky, we distinguish three classes of $\gamma$-ray blazar candidates on the basis of their $s_{b}$ and/or $s_{q}$ values:

- class A: WISE sources with $0.24<s_{b}<1.00$ and $0.38<s_{q}<1.00$;

- class B: WISE sources with $0.24<s_{b}<1.00$ or $0.38<s_{q}<1.00$;

- class C: WISE sources with $0.10<s_{b}<0.24$ and $0.14<s_{q}<0.38$.

All the WISE sources with $s_{b}<0.10$ or $s_{q}<0.14$ are considered outliers of the WGS. Sources of class A are the rarest with respect to the other classes (Paper IV).

Our association procedure consists in ranking all the WISE sources within the searching region of an unidentified $\gamma$-ray source as described above and indicating as a $\gamma$-ray blazar candidate the positionally closest source belonging to the highest class. Our association procedure provides a completeness of $87 \%$ based on the a posteriori re-association of the ROMA-BZCAT blazars, detected by WISE and associated with Fermi-LAT sources.

\section{4. $\quad \gamma$-ray blazar candidates among the unidentified INTEGRAL sources}

We applied our new association method to the case of the 86 UISs selected above. This process allows us to verify if there is a $\gamma$-ray blazar candidate within the positional error region of each UIS analyzed.

We considered a searching region with radius equal to the position error at $90 \%$ confidence level, as reported in the 4IC catalog; then, we estimated the IR WISE colors for all the sources that lie within the searching region.

Running our association procedure, we found that 68 out of 86 UISs have only outliers of the WGS lying in their searching regions, while within the remaining 18 UISs we found $4 \gamma$-ray 
blazar candidates of class A, 12 of class B and 2 of class C. In Table 1, we present the list of $\gamma$-ray blazar candidates found for the 18 UISs together with their IR colors, as well as the $s_{b}$ and $s_{q}$ parameters. We also estimated the probability to find a generic WISE source with the same $s$ values in 36 random circular regions of the WISE sky having the same radius $R$ of the searching regions. We found that this is smaller than $10^{-4}$. We note that the positional accuracy of the UISs is a least order of magnitude better than that of the unidentified $\gamma$-ray sources in 2FGL.

Summarizing our results, we found 18 WISE $\gamma$-ray blazar candidates that could be candidate counterparts of the corresponding UISs responsible for the hard X-ray emission detected by INTEGRAL.

\section{5. $\quad \gamma$-ray blazar candidates observed by Swift}

We found that among the $\gamma$-ray blazars selected according to our association procedure, 10 candidates out of 18 have at least one Swift pointed observation. In addition, none of these $\gamma$ ray blazar candidates has a $\gamma$-ray counterpart in the 2FGL. We reduced and analyzed these Swift observations to verify if these WISE $\gamma$-ray blazar candidates have an optical-UV or soft X-ray counterpart. Here we report the data reduction and analysis procedures used in our Swift data analysis. The comparison between the Swift and the WISE images will be presented in Section 6 ,

\subsection{UVOT data analysis}

We followed the same UV-Optical Telescope (UVOT) reduction procedure described in (Tramacere et al. 2007; Massaro et al. 2008a) consequently, here we report only the basic details.

Several filter combinations are available for UVOT observations; however, we note that not all the optical and UV data are available for each source. The detection algorithm UVOTDETECT was used to confirm the presence of the optical-UV counterpart of the $\gamma$-ray blazar candidates. We then performed the photometric analysis using the UVOTSOURCE tool. Counts were extracted from a $6^{\prime \prime}$ radius aperture in the $V, B$, and $U$ filters and from a $12^{\prime \prime}$ radius aperture for the other UV filters ( $U V W 1, U V M 2$, and $U V W 2)$, to properly take into account the wider Point Spread Function in these bandpasses. The count rate was corrected for coincidence loss, and the background subtraction was performed by estimating its level in an offset circular region at $20^{\prime \prime}$ from the source.

The correction for the interstellar reddening was obtained assuming the $E(B-V)$ values from Schlegel et al. (1998) and the corrections described in Cardelli et al. (1989), while the fluxes 
were derived with the same conversion factors given by Giommi et al. (2006).

\subsection{XRT data analysis}

The X-Ray Telescope (XRT) data reduction used in the following is also the same one described in (Tramacere et al. 2007; Massaro et al. 2008b; Massaro et al. 2011c); here we only report the basic details.

The XRT data analysis has been performed with the XRTDAS software (v. 2.1), developed at the ASI Science Data Center (ASDC) and distributed within the HEAsoft package (v. 6.10.0).

Event files were calibrated and cleaned with standard filtering criteria using the XRTPIPELINE task, combined with the latest calibration files available in the Swift $C A L D B$ distributed by HEASARC. Only events in the energy range $0.3-10 \mathrm{keV}$ with grades $0-12$ were used. When more than a single Swift pointing of each source has been performed and is available within the Swift archive, we combined several low S/N observations, because the the co-added X-ray image increases significantly the source detection. No signatures of pile-up were found in our XRT observations.

Given the low exposure of the Swift observations it was not possible to carry out a detailed spectral analysis, so unless stated otherwise we used the detection algorithm detect, a tool of the $X I M A G E$ package for all the Swift observations. The detect algorithm locates the X-ray point sources using a sliding-cell method taking into account the average background intensity. The position and intensity of each detected source is calculated using a box whose size maximizes the signal-to-noise ratio. This detection algorithm has been extensively used in the Swift serendipitous survey in deep XRT gamma-ray burst fields (see also Puccetti et al. 2011, for additional details). Statistical and systematic uncertainties on count rates are added quadratically.

Finally, we measured the net count rates for each detected soft X-ray source and we converted them into fluxes assuming a power-law spectrum with spectral index 1 and using WEBPIMMS 4 .

\section{Results on the Swift analysis}

Applying our new association procedure developed for the unidentified $\gamma$-ray sources of Fermi-LAT to the UISs, we found that 18 sources out of the 86 analyzed have a $\gamma$-ray blazar candidate as possible counterparts. We note that this new association method proposed for the

\footnotetext{
${ }^{4}$ http://heasarc.nasa.gov/Tools/w3pimms.html
} 
unidentified $\gamma$-ray sources of Fermi-LAT does not have the same efficiency when applied to soft $\gamma$-rays and/or hard X-rays. In fact, in the Fermi-LAT energy range (i.e., $30 \mathrm{MeV}-10 \mathrm{GeV}$ ) blazars are the largest known $\gamma$-ray population (Nolan et al. 2012), while the hard X-ray band is generally dominated by the emission of different classes of AGN, such as Seyfert galaxies, which constitute $\sim 24 \%$ in comparison with the $2.4 \%$ for blazars already associated in the 4IC. This implies that the WISE $\gamma$-ray blazar candidates are not necessarily the low-energy counterparts of the UISs.

The relation between the IR spectral shape in the WISE energy range and that in the $\gamma$-rays is based on our association method (e.g., Paper II). On the other hand, in hard X-rays there is not yet evidence of a correlation between the IR and the X-ray emission of blazars, thus the eventual association between the WISE $\gamma$-ray blazar candidate and the UISs is less robust that in the case of the Fermi-LAT sources.

For 10 out of the 18 WISE $\gamma$-ray blazar candidates, we also found optical-UV and X-ray observations available in the Swift archive that could be helpful to verify if they are the low-energy counterparts of the UISs. We found that 7 out of the 10 WISE $\gamma$-ray blazar candidates in the Swift archive have a clear counterpart in X-rays and in the optical-UV bands, showing a typical Spectral Energy Distribution (SED) dominated by non-thermal emission, as for the two cases shown in Figure 1, where J035651.52+624553.8 has also a radio counterpart at 1.12 arcsec from the WISE position. In Table 1, we report the INTEGRAL name together with the WISE $\gamma$-ray blazar candidates, the J2000 coordinates RA and DEC, the distance between the WISE source and the INTEGRAL position in arcsec, the WISE colors (i.e., $c_{12}=[3.4]-[4.6], c_{23}=[4.6]-[12]$, $\left.c_{23}=[12]-[22]\right)$, the $s_{b}$ and the $s_{q}$ derived from our WGS method, the Swift UVOT detections and the Swift XRT detections with the X-ray counts in the soft (0.3-1 keV) and in the hard (i.e., 1-10 $\mathrm{keV}$ ) bands together with the X-ray hardness ratio $H R$ derived from the net number of counts.

For remaining three WISE $\gamma$-ray blazar candidates we did not find a clear counterpart in Swift observations. This result could be due to the short exposures of the archival observations.

However, we note that in the above 10 candidates, the Swift XRT flux is not in agreement with the extrapolation of the INTEGRAL spectrum, which is generally one order of magnitude larger than the Swift XRT estimate. This discrepancy is not sufficient to exclude the blazar association of the UISs because blazars exhibit rapid X-ray variability; however, the it could suggest that the blazar is not the most probable low-energy counterpart for the UISs, in agreement with the fact that they are not the dominant class of AGN in the hard X-rays. We note that the $\gamma$-ray blazar candidates found with our method are WISE sources, detected in all four WISE bands, in particular at $22 \mu \mathrm{m}$ as the case of IGRJ14549-6459 shown in Figure 2, for which the WISE candidate counterpart appear to have the IR colors of blazars.

In Figure 3 and Figure 4, we also show the comparison between the soft X-ray and the optical- 


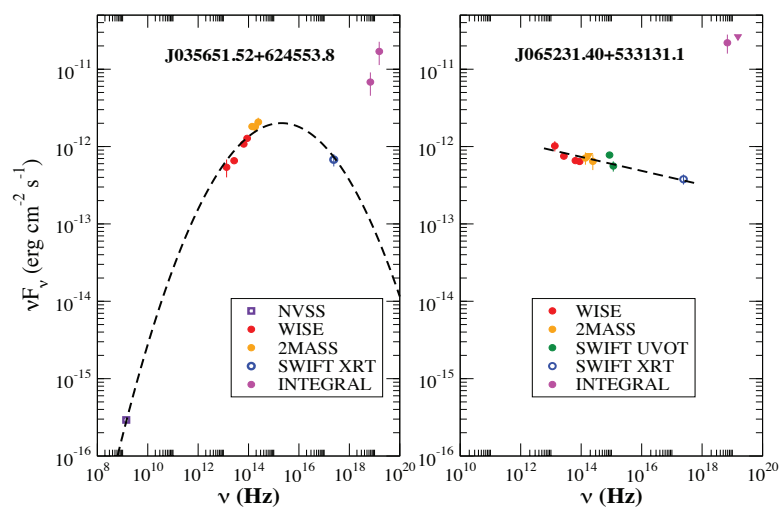

Fig. 1. - The SEDs of two example of WISE $\gamma$-ray blazar candidates: J035651.52+624553.8 and J065231.40+533131.1. The dashed line in the left panel is the typical log-parabolic model adopted to describe the non-thermal SED of J035651.52+624553.8 while in the case of J065231.40+533131.1 a simple power-law, over 6 orders of magnitude, has been used. As described in Section 6 there is discrepancy between the XRT fluxes and those of INTEGRAL that do not support the blazar association of the UIS.

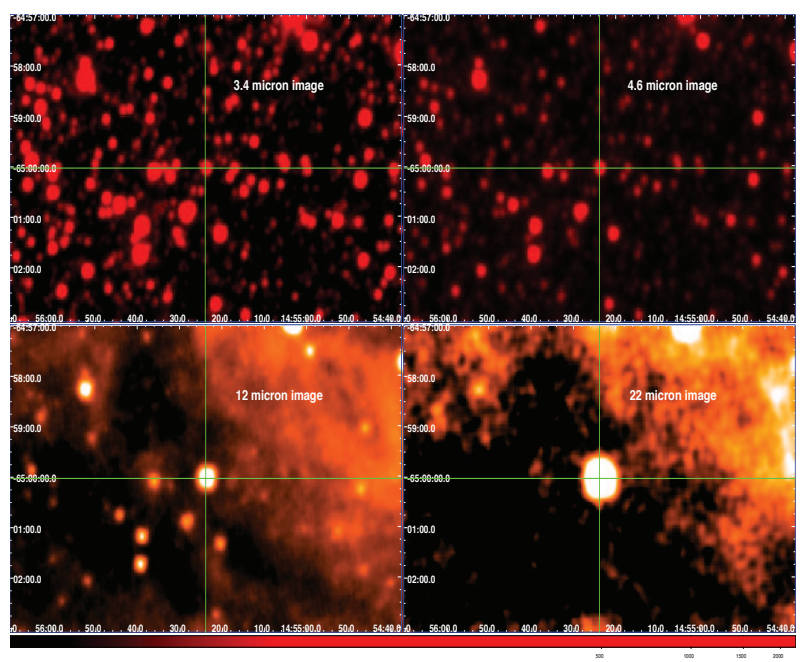

Fig. 2.- The WISE IR images at $3.4 \mu \mathrm{m}, 4.6 \mu \mathrm{m}, 12 \mu \mathrm{m}, 22 \mu \mathrm{m}$, respectively for the Field of View (FOV) for IGRJ14549-6459, centered on the position of the $\gamma$-ray blazar candidate. The WISE $\gamma$-ray blazar candidate is highlighted with the green cross in the center of the WISE images. It is clear that the source selected with our association method is the only one detected in all 4 WISE bands. 
UV images of Swift with the WISE IR data, for the FOV of two examples of UISs centered on the positions of our $\gamma$-ray blazar candidates: IGRJ06523+5334 and IGRJ13045-5630, respectively.

\section{Summary}

We applied our new association method successfully used for the unidentified $\gamma$-ray sources in the 2FGL to the UISs to test if it is possible to find WISE blazar counterparts at low energies responsible for the hard X-ray emission detected by INTEGRALwithin the searching regions of the UISs.

We found that 18 out of 86 UISs analyzed clearly have a blazar counterpart within the searching regions, and for 10 of them also Swift archival observations are available. However, for the latter 10 sources in Swift we did not find a good agreement between the Swift X-ray flux and the one estimated by the extrapolation of the INTEGRAL spectrum. Thus, we are not able to confirm if the WISE $\gamma$-ray blazar candidates found with our method could be associated with the UISs considered.

On the other hand, one crucial result arises from our analysis. We found that the WISE $\gamma$-ray blazar candidates selected from our method in these serendipitous Swift observations of the UIS fields of view have clear optical and/or UV and soft X-ray counterparts. This is strongly in agreement with the expectations driven by their blazar nature. It is worth noting that J035651.52+624553.8 has also a radio counterpart and in addition, the SEDs of these WISE $\gamma$-ray blazar candidates are in agreement with a non-thermal shape over several orders of magnitude.

Finally, we remark that future follow up observations, in particular spectroscopic optical data, are necessary to clarify the nature of the WISE $\gamma$-ray blazar candidates and consequently the nature of the UISs (see e.g., Masetti et al. 2008; Masetti et al. 2009; Masetti et al. 2010).

We thank the anonymous referee for the his/her comments. We are grateful to D. J. Thompson for all his comments helpful toward improving our presentation. F. Massaro is grateful to H. Smith, J. Grindlay, M. Ajello, E. Bottaccini for their helpful discussions. The work at SAO is supported in part by the NASA grant NNX10AD50G and NNX10AD68G. R. D'Abrusco gratefully acknowledges the financial support of the US Virtual Astronomical Observatory, which is sponsored by the National Science Foundation and the National Aeronautics and Space Administration. TOPCAT 5 (Taylor 2005) and SAOImage DS9 were used extensively in this work. Part of this work is based

\footnotetext{
${ }^{5}$ http://www.star.bris.ac.uk/ mbt/topcat/
} 
on archival data, software or on-line services provided by the ASI Science Data Center. This publication makes use of data products from the Wide-field Infrared Survey Explorer, which is a joint project of the University of California, Los Angeles, and the Jet Propulsion Laboratory/California Institute of Technology, funded by the National Aeronautics and Space Administration.

\section{REFERENCES}

Baumgartner, W. H. et al. 2010 ApJS submitted

Bird, A. J. et al. 2010 ApJS, 186, 1

Cardelli, J. A., Clayton, G. C., Mathis, J. S. 1989 ApJ, 345, 245

Cusumano, G. et al. 2010 A\&A, 524A, 64

Cutri, R. M. 2011, wise.rept, 1

D’Abrusco, R., Massaro, F., Ajello, M., Grindlay, J. E., Smith, Howard A. \& Tosti, G. 2012 ApJ accepted

Giommi, P. et al. 2006 A\&A, 456, 911

Lebrun, F. et al. 2003, A\&A, 411, L141

Masetti, N. et al. 2008 A\&A, 482, 113

Masetti, N. et al. 2009 A\&A, 495, 121

Masetti, N. et al. 2010 A\&A, 519A, 96

Massaro, F., Giommi, P., Tosti, G., Cassetti, A., Nesci, R., Perri, M., Burrows, D. \& Gehrels, N. 2008 A\&A, 489, 1047

Massaro, F., Tramacere, A., Cavaliere, A., Perri, M., Giommi, P. 2008 A\&A, 478, 395

Massaro, E., Giommi, P., Leto, C., Marchegiani, P., Maselli, A., Perri, M., Piranomonte, S., Sclavi, S. 2009 A\&A, 495, 691

Massaro, E., Giommi, P., Leto, C., Marchegiani, P., Maselli, A., Perri, M., Piranomonte, S., Sclavi, S. 2010 http://arxiv.org/abs/1006.0922

Massaro, F., D’Abrusco, R., Ajello, M., Grindlay, J. E. \& Smith, H. A. 2011 ApJ, 740L, 48 
Massaro, E., Giommi, P., Leto, C., Marchegiani, P., Maselli, A., Perri, M., Piranomonte, S., "Multifrequency Catalogue of Blazars (3rd Edition)", ARACNE Editrice, Rome, Italy

Massaro, F., Paggi, A., Elvis, M. \& Cavaliere, A. 2011 ApJ, 739, 73

Massaro, F., D’Abrusco, Tosti, G., R., Ajello, M., Gasparrini, D., Grindlay, J. E. \& Smith, H. A. 2012a ApJ submitted

Massaro, F., D’Abrusco, Tosti, G., R., Ajello, M., Paggi, A., Gasparrini, D. 2012b ApJ submitted

Massaro, F. et al. ApJ in preparation

Nolan, P. L. et al. 2012 ApJS, in press

Puccetti, S. et al. 2011 A\&A, 528, A122

Schlegel, D, J., Finkbeiner, D. P., Davis, M. 1998 ApJ, 500, 525

Taylor, M. B. 2005, ASP Conf. Ser., 347, 29

Tramacere, A. et al. 2007 A\&A, 467, 501

Ubertini, P. et al. A\&A, 411, L131

Wright E. L., et al. 2010 AJ, 140, 1868

This preprint was prepared with the AAS LATEX macros v5.2. 


\begin{tabular}{|c|c|c|c|c|c|c|c|c|c|c|c|c|}
\hline $\begin{array}{l}\text { INTEGRAL } \\
\text { name }\end{array}$ & $\begin{array}{l}\text { WISE } \\
\text { name }\end{array}$ & $\begin{array}{l}\text { RA } \\
(\mathrm{deg})\end{array}$ & $\begin{array}{l}\text { DEC } \\
(\mathrm{deg})\end{array}$ & $\begin{array}{c}\text { distance } \\
\text { arcsec }\end{array}$ & $c_{1}$ & $c_{2}$ & $c_{3}$ & $s_{b}$ & $s_{q}$ & $\begin{array}{c}\text { UVOT/ XRT } \\
\text { detec. }\end{array}$ & $\begin{array}{c}\text { counts } \\
0.3-1 \mathrm{keV}\end{array}$ & $\begin{array}{c}\text { counts } \\
1-10 \mathrm{keV}\end{array}$ \\
\hline \multicolumn{13}{|l|}{ class A sources } \\
\hline IGRJ04442+0450 & J044415.86+045126.6 & 71.07 & 4.86 & 88.17 & $1.17(0.03)$ & $3.06(0.03)$ & $2.81(0.05)$ & 0.43 & 0.75 & & - & - \\
\hline IGRJ06523+5334 & J065231.40+533131.3 & 103.13 & 53.53 & 219.42 & $1.02(0.04)$ & $3.01(0.06)$ & $2.45(0.13)$ & 0.30 & 0.43 & $\mathrm{n} / \mathrm{y}$ & 18 & 41 \\
\hline IGRJ14549-6459 & $\mathrm{J} 145523.80-650002.5$ & 223.85 & -65.00 & 212.20 & $1.09(0.03)$ & $2.71(0.03)$ & $2.43(0.03)$ & 0.91 & 0.92 & $\mathrm{n} / \mathrm{y}$ & & 38 \\
\hline IGRJ16413-4046 & J164122.31-404714.5 & 250.34 & -40.79 & 28.13 & $0.73(0.04)$ & $2.05(0.03)$ & $1.36(0.03)$ & 0.94 & 0.39 & $\mathrm{n} / \mathrm{n}$ & - & - \\
\hline \multicolumn{13}{|l|}{ class B sources } \\
\hline IGRJ03502-2605 & J035018.94-260423.6 & 57.58 & -26.07 & 116.77 & $1.12(0.04)$ & $2.51(0.07)$ & $2.33(0.22)$ & 0.26 & 0.31 & $\mathrm{n} / \mathrm{y}$ & 10 & 2 \\
\hline IGRJ03564+6242 & $\mathrm{J} 035651.52+624553.8$ & 59.21 & 62.76 & 264.69 & $0.87(0.04)$ & $2.40(0.06)$ & $1.93(0.22)$ & 0.35 & 0.17 & $\mathrm{n} / \mathrm{y}$ & 19 & 12 \\
\hline IGRJ07225-3810 & J072228.14-381457.6 & 110.62 & -38.25 & 293.05 & $1.06(0.05)$ & $2.68(0.09)$ & $1.89(0.20)$ & 0.26 & 0.24 & & & 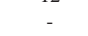 \\
\hline IGRJ13045-5630 & J130431.77-563058.5 & 196.13 & -56.52 & 58.56 & $0.94(0.03)$ & $3.30(0.03)$ & $2.71(0.03)$ & 0.00 & 0.69 & $\mathrm{n} / \mathrm{y}$ & 4 & 40 \\
\hline IGRJ13107-5626 & J131037.06-562654.3 & 197.65 & -56.45 & 29.06 & $1.29(0.03)$ & $2.78(0.03)$ & $2.26(0.04)$ & 0.00 & 0.84 & - & - & - \\
\hline IGRJ15293-5609 & $\mathrm{J} 152900.40-560830.4$ & 232.25 & -56.14 & 149.87 & $0.82(0.06)$ & $2.44(0.06)$ & $2.39(0.07)$ & 0.43 & 0.29 & - & - & - \\
\hline IGRJ15311-3737 & $\mathrm{J} 153051.78-373457.1$ & 232.72 & -37.58 & 211.38 & $0.87(0.03)$ & $2.17(0.03)$ & $2.05(0.06)$ & 0.70 & 0.29 & $y / y$ & 45 & 220 \\
\hline IGRJ16560-4958 & J165551.96-495732.3 & 253.97 & -49.96 & 59.51 & $0.75(0.05)$ & $2.28(0.04)$ & $2.05(0.08)$ & 0.52 & 0.22 & - & - & - \\
\hline IGRJ17314-2854 & $\mathrm{J} 173111.38-285701.8$ & 262.80 & -28.95 & 180.76 & $0.34(0.03)$ & $1.25(0.02)$ & $0.88(0.03)$ & 0.00 & 0.44 & - & - & - \\
\hline IGRJ17448-3232 & $\mathrm{J} 174440.89-323155.8$ & 266.17 & -32.53 & 89.35 & $0.64(0.04)$ & $1.88(0.03)$ & $1.19(0.05)$ & 0.45 & 0.30 & $\mathrm{n} / \mathrm{n}$ & & - \\
\hline IGRJ19552+0044 & $\mathrm{J} 195504.07+004421.0$ & 298.77 & 0.74 & 106.47 & $1.04(0.05)$ & $2.72(0.08)$ & $2.18(0.25)$ & 0.29 & 0.29 & - & - & - \\
\hline IGRJ20450+7530 & $\mathrm{J} 204522.41+753057.4$ & 311.34 & 75.52 & 90.73 & $0.87(0.04)$ & $2.29(0.08)$ & $2.30(0.29)$ & 0.27 & 0.16 & $y / y$ & 17 & 52 \\
\hline \multicolumn{13}{|l|}{ class $\mathrm{C}$ sources } \\
\hline IGRJ13550-7218 & & 18 & -72.24 & & & & & 0.17 & 0.22 & $\mathrm{n} / \mathrm{n}$ & - & - \\
\hline IGRJ16388+3557 & $\mathrm{J} 163901.61+355510.7$ & 249.76 & 35.92 & 200.60 & $1.07(0.05)$ & $2.75(0.10)$ & $2.64(0.26)$ & 0.19 & 0.23 & - & & - \\
\hline
\end{tabular}

Col. (1) INTEGRAL name

Col. (2) WISE blazar candidates

Cols. $(3,4)$ the J2000 coordinates RA and DEC

Col. (5) the distance between the WISE source and the INTEGRAL position in arcseconds

Cols. (6,7,8) the WISE colors (i.e., $\left.c_{1}=[3.4]-[4.6], c_{2}=[4.6]-[12], c_{2}=[12]-[22]\right)$; the $1 \sigma$ errors are reported in parenthesis.

Cols. $(9,10)$ the $s_{b}$ and the $s_{q}$ derived form our WGS method

Cols. (11,12) the Swiff UvOT detocions and the Swift XRT detections

Cols. $(13,14)$ the X-ray counts in the soft $(0.3-1 \mathrm{keV})$ and in the hard (i.e., $1-10 \mathrm{keV})$ band, respectively 


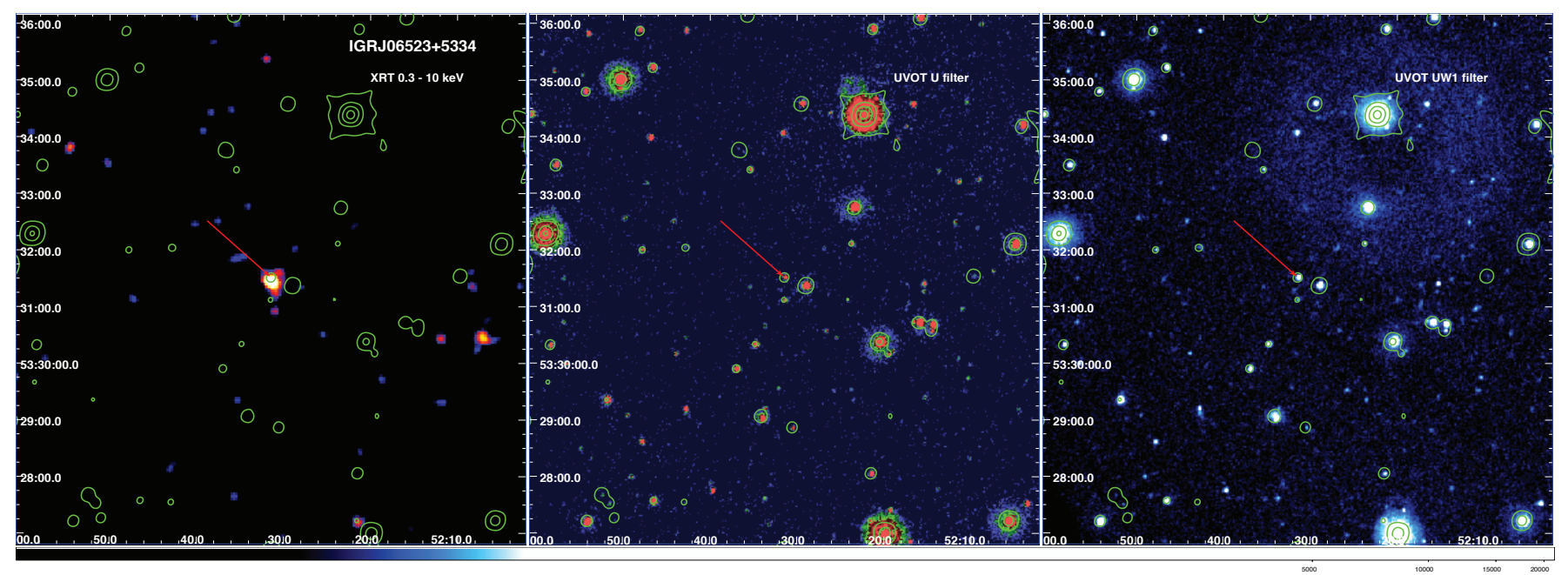

Fig. 3.- The WISE $3.4 \mu \mathrm{m}$ IR contours (green) overlaid on the Swift optical-UV and X-ray images, for the FOV of IGRJ06523+5334, centered on the position of the selected WISE blazar candidate. It is clear that the WISE blazar candidate (red arrow) has a clear counterpart in the soft $\mathrm{X}$-rays and in the optical-UV bands.

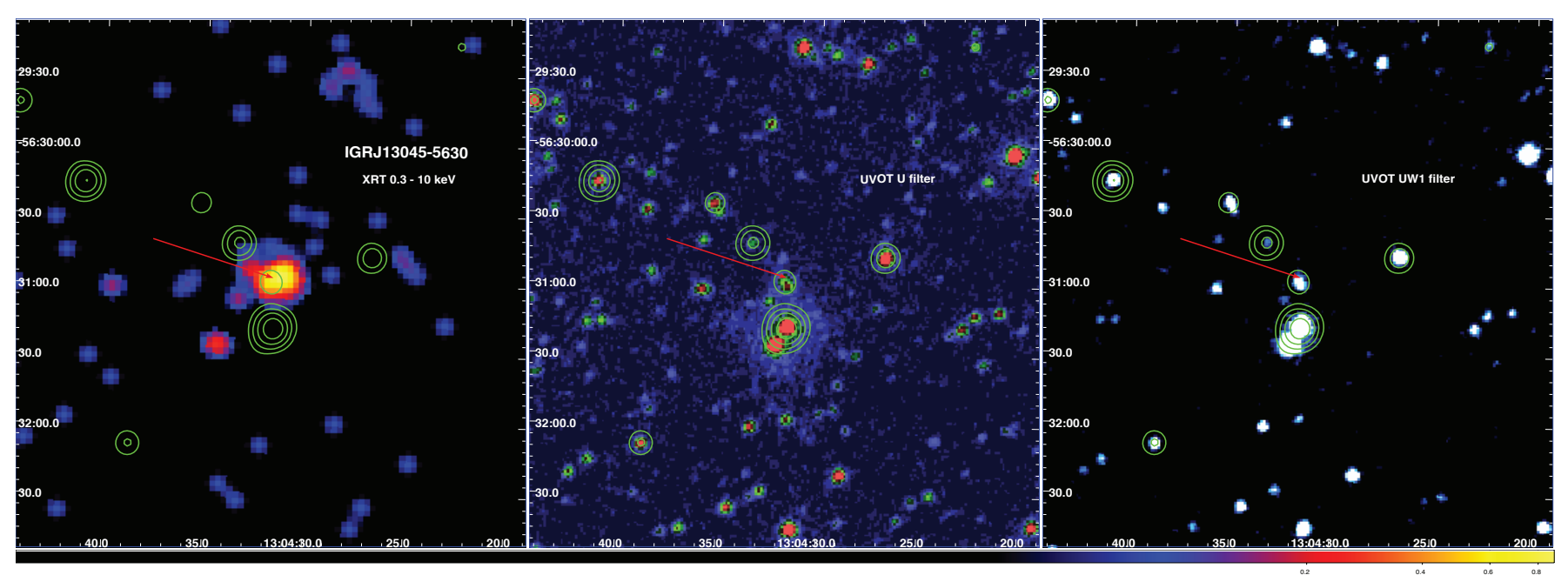

Fig. 4.- Same of Figure 3 for the IGRJ 13045-5630 FOV. 